\title{
Influencing factors of urban innovation and development: a grounded theory analysis
}

\author{
Jing-Xiao Zhang ${ }^{1}$ D . Jia-Wei Cheng ${ }^{1}$. Simon Patrick Philbin ${ }^{2}$. \\ Pablo Ballesteros-Perez ${ }^{3}$. Martin Skitmore ${ }^{4} \cdot$ Ge Wang $^{5}$
}

Received: 18 May 2021 / Accepted: 14 January 2022 / Published online: 2 February 2022

(c) The Author(s), under exclusive licence to Springer Nature B.V. 2022

\begin{abstract}
Urban innovation and development are a core driver for promoting the industrial, economic, and social development of cities. However, the factors that affect the innovation and development of cities lack systematic analysis as well as interaction analysis. Based on a multidimensional perspective, this study suggests that natural, economic, and social factors are three major factors conditioning urban innovation and development. A grounded theoretical qualitative method is further adopted to code relevant research literatures, news reports and interview materials, resulting in an onion factors model. We find that natural factors-including environmental quality, geographic location, and city scale-are prerequisite for conditioning urban innovation and development. Economic factors are also key, including economic level, industrial structure, industrial agglomeration, and technological innovation. Social factors are guarantee factors, including administrative hierarchy, cultural environment, population structure, and government management and services, i.e., they are essential for cities to become adaptable in the current dynamic situation. The study provides theoretical support and practical directions for the formulation of policies for urban innovation development.
\end{abstract}

Keywords Urban innovation and development $\cdot$ Social-economic-natural complex ecosystem theory $\cdot$ Grounded theory $\cdot$ Influencing factors

\section{Introduction}

Urban innovation and development are a key driver underpinning the performance of innovation systems at both regional and national levels (Ari-Veikko, 2016, Lauer \& Liefner, 2019, Deng \& Chen, 2020). With the world facing the global economic, environmental, and social disruption of the "post-epidemic era", there is an urgent need to ensure urban environments are constantly improved. One way of achieving this is by systematically adopting innovation systems and technologies in our cities. This urban innovation and development

Jing-Xiao Zhang

zhangjingxiao964@126.com

Extended author information available on the last page of the article 
has significant capacity to facilitate the transition to a more sustainable future as well as steer economic growth patterns leaning on regional development (Popescu, 2020).

From a global perspective, many companies and governments are shifting their focus from solely capital provision to innovation systems. The core feature of this emerging economy is moving from a 'global production network' to a 'global innovation network', that is, from cities considering not just their economic strength, but also their innovation capabilities (Li \& Zhang, 2020). In this respect, according to Innovation Cities Index 2019: Global, the ranking of Chinese cities has increased significantly. However, the ten most innovative cities are still from the United States, Japan, United Kingdom, France, Singapore, and Canada (2thinknow, 2019). Yet, China has made continuous efforts for urban innovation development since their 2008 innovative city pilot plan (Zheng et al., 2019). Unfortunately, China's urban innovation development capabilities have an unbalanced development pattern that is producing different regional results. This cannot be simply explained by the scale effect associated with regional development (Njøs \& Jakobsen, 2016). Arguably, they must be related to specific factors, such as access to human capital, technology, and other resources. Therefore, clarification of the key factors that contribute to urban innovation is still needed to improve the overall innovation and development level of cities in China and other countries.

Hence, it is unsurprising that the study of urban innovation and development is attracting much more research attention. Disciplines such as economics, geography, demography, and environmental science now regard urban innovation and development as an important area of research. Some scholars have produced noticeable research into the innovative development of special types of cities (e.g., resource-based cities) (Tan et al., 2014), municipalities under control of the central government ( $\mathrm{Li} \& \mathrm{Wu}, 2015$ ), and old industrial cities (Dong \& Jing, 2014). Yong et al. (2016) also adopt the informatization development perspective and analyze the effects of financial and technological innovation development. Liu et al. (2016) investigate prefecture-level cities in China to analyze the relationship between their level of urbanization and urban innovation and development. More recently, Yang et al. (2019) analyze the relationship between industrial development, urban expansion, and urban innovation development from the perspective of industry-city integration.

As a result, due to the different disciplinary backgrounds and research perspectives involved, studies provide different understandings of the concept of urban innovation and development. Hence, the present study defines urban innovation and development as an urban development model aimed at promoting sustainable and coordinated economic growth by leveraging innovation. This goal is achieved as the city, a complex system, gradually adjusts and self-balances through collaboration and integration of innovative-based resources.

Clarifying the influencing factors of urban innovation and development is the core and foundation to improve the overall innovation and development level of the city (Elkington, 1998; Alhaddi, 2015). As highlighted earlier, various studies have focused on different aspects of urban innovation and development. However, they are often limited to a relatively narrow research scope when interpreting the complex phenomenon of how an urban environment undergoes the process of development. Adopting a limited research perspective undoubtedly allows a detailed analysis of some specific innovation and urban-related factors development. However, it may not be representative of the overall mechanisms associated with urban innovation and development. In addition, research in recent years has mainly focused on the establishment of index systems to evaluate the innovation effectiveness of cities, and has paid less attention to the influencing factors of urban innovation and development and their interaction. This way, although the innovation and development 
effectiveness of different cities is accurately measured, the existing deficiencies of urban innovation and development cannot be effectively addressed.

Therefore, focusing on the core issue of "what factors affect urban innovation and development, and how these factors affect urban innovation and development", this study takes the urban system as the research object. It also carries out a comprehensive research on the influencing factors of urban innovative development from the perspectives of ecological environment and social development. And by doing so, it integrates existing research on urban innovative development. Based on the qualitative research method of grounded theory, this study identifies those factors affecting urban innovation and development in the existing literature, including news reports and interview materials. Then, it systematically analyzes the influencing factors of urban innovation and development, and defines the interaction between influencing factors. As a result, the main significance of this research lies in:

(1) This research does not require delving in the context of urban innovation and development, but still analyzes urban innovation and development from three perspectives: nature, society and economy. Then, it constructs a theoretical model of the factors affecting urban innovation and development, and by doing so, it expands the research scope of compound ecosystem theory in the context of urban innovation and development.

(2) This research also provides theoretical support, revised directions and practical entry points for policy-making of relevant departments, and a new perspective on current problems (e.g., population growth, resource depletion, and environmental pollution) many cities currently suffer. It also promotes urban innovative development in consideration of the triple bottom line of economic, environmental, and social development.

\section{Background}

\subsection{The urban innovation system}

At the beginning of the twentieth Century, Schumpeter proposed the concept of "innovation" in the book Theory of Economic Development. He began to nurture the theory of innovation mostly based on production technology methods and changes in the process of economic growth (Schumpeter \& Joseph, 1947). In the late 1980s, innovation research moved toward the so-called "systems paradigm". A large number of concepts emerged, which can be roughly divided in two categories: the first encompasses the spatial and geographic characteristics of innovation systems; the second analyzes the theory of innovation systems from the characteristics of industrial technology (Zhou et al., 2012). Both national and regional innovation systems focus on the spatial and geographic form of innovation, while the industrial and technological innovation system emphasizes the technical characteristics of innovation. However, current research into urban innovation systems continues on the trajectory of regional innovation systems (Simmie \& Sennett, 1999).

In the case of China, Zhao and Li (2003) analyzed the country's regional economic development, employing innovation theory, regional economic theory, and technology diffusion theory to propose a theory of urban innovation. Their study highlighted that urban innovation refers to the socio-economic system formed by the interdependence and interaction of various innovation-related elements. These elements include innovative institutions 
and organizations (such as enterprises, governments, universities, scientific research institutions, and intermediary organizations), non-subject elements (i.e., materials and resources necessary for innovation), together with institutions and policies that coordinate the relationship between these elements within the city region. However, Sui (2004) views the urban innovation system from a different perspective, conceiving the city as an innovation ecosystem where the diffusion effect of urban innovation, social structure, and the clustering effect of science and technology industries are all interdependent. As a system, urban innovation not only has the overall system's integrity, relevance, structure, dynamics, purpose, and environmental adaptability, but also has complex (and counter-intuitive) policy change resistance and other system characteristics in addition to its socio-economic systemic nature (Zhao \& Hu, 2010).

Urban innovation systems can also be viewed as complex systems composed of many interrelated sub-systems and artefacts, which means that the urban innovation system boundaries must be clearly defined before the factors that influence such a system can be studied. In this regard, Hou et al. (2009) add knowledge innovation ability and environmental innovation ability, thereby expanding urban innovation to six elements. Chen and $\mathrm{Xu}$ (2009) analyze the innovation experience of innovative cities in several countries, finding that urban innovation systems have three basic elements: innovation subject, innovation resources, and innovation environment. These studies also discuss the operating mechanisms of innovation systems, while Markatou and Alexandrou (2015) note that a small-scale innovation system has to consider not only economic factors but also the whole spectrum of societal challenges.

As a result, current studies generally agree that urban innovation systems are composed of several components, generally named as main elements, functional elements, and environmental elements (Zhou et al., 2012; Markatou \& Alexandrou, 2015). Main elements are the innovation institutions and organizations connected with innovation. These include enterprises, scientific research institutions, and universities. The functional elements are the systems and policies that coordinate the relationships between the elements, including urban public services, governmental governance mechanisms, and the financial environment and systems. Environmental elements are the elements related to urban natural ecological aspects. These include the urban geographic location, materials and resources required for innovation, and environmental quality. Therefore, the objective of the present research is to conduct a bibliographic study to identify the constituent elements of an urban innovation system, and then evaluate the factors influencing urban innovation and development.

\subsection{Complex ecosystem theory}

Ecological theory is considered one of mankind's scientific foundations that seeks to solve both major and contemporary social problems (Nielsen, 2007). Achieving the innovative and sustainable development of a city requires addressing a series of problems associated with its environment together with those related to the livelihood and social position of its inhabitants (e.g., rapid urban population growth, resource shortages, rising emissions and pollution, and ecological imbalances) (Pickett et al., 2001; Marzluff et al., 2008; Viezzer \& Biondi, 2021).

In response to an increasing number of major social problems, Ma (1984) observes that the challenges of social development cannot be regarded as standalone social problems, economic problems, or natural problems. Instead, they must be treated as complex 
problems that can only be addressed from a systems level perspective, based on which he proposed the socio-economic-natural complex ecosystem viewpoint. In this framework, human social, economic, and environmental activities cannot go beyond the "boundaries" of this composite system to promote sustainability-the essence of sustainable development. As a miniature ecological landscape on the surface of the planet, cities frequently suffer from high-intensity social, economic, and natural agglomeration effects. They also suffer from large-scale and concentrated population areas, resource scarcities, and environmental impacts. In this scenario, the innovative development of cities can be assimilated to one of the pathways for achieving sustainable development goals (Kumari, 2014; Coscieme et al., 2021)-most likely one of the reasons why research in this area continues to grow.

Major research areas in this domain include investigating the effects of urban ecological environment caused by urbanization; the balanced development of urban society, economy, and the environment; as well as sustainable, ecological, and healthy cities. For example, He et al. (2013) discuss the regulation mechanism of the coordinated development of urban economy and environment; Wang et al. (2014) suggest that the foundations of urban composite ecological management are based on the theory of complex ecosystems, viz. a harmonious society, circular economy, and safe ecology; and Scalia et al. (2020) construct a dynamic system based on ecological and economic coupled variables.

Furthermore, the development of systems science provides a scientific method for obtaining a comprehensive understanding of complex matters with a holistic approach. Indeed, a general consensus is the need to examine cities and urban centers from multiple angles (Eskov et al., 2012). The theory of compound ecosystems regards human society as a type of artificial ecosystem that is dominated by human behavior. The latter, though, is supported by the natural environment and social systems. This takes into account the economic issues, social issues, and environmental issues involved in the process of urban development. Consequently, this approach poses an ideal theoretical platform to study urban innovation and development from a broader systems perspective. In this respect, the development and changes of the various sub-systems in the composite ecosystem are restricted by different laws. For example, the sub-systems are interdependent through material circulation, energy flow, and information transmission. The urban innovation system also includes socio-economic attributes and human culture as well as biology and the environment-implying that, in addition to natural laws, there are also economic and social laws regulating the system (Markatou \& Alexandrou, 2015; Jagt et al., 2020). Hence, the core idea of compound ecosystem theory is to reveal the unity of human society and the natural environment. This provides three perspectives for research into urban innovation and development-namely, the natural, social, and economic perspectives.

\section{Research design}

\subsection{Research methods}

Research into the influencing factors of urban innovation and development is relatively fragmented, with a particular lack of comprehensive and systematic study. Researchers from various disciplines have different opinions concerning the influencing factors of urban innovation and development, leading to significant differences in how this phenomenon is understood. In situations when it is difficult to identify and determine the scope of influencing variables, it is more difficult to design suitable measurement scales 
or even use quantitative analysis. Hence, an exploratory and deductive investigation enquiry, such as a qualitative research method, is needed.

Grounded theory was first introduced by two sociologists, Barney Glaser and Anselm Strauss, in their seminal 1967 book, The Discovery of Grounded Theory. Grounded theory is a research path, not a physical "theory". The purpose of this form of research is to generate a theory, and the theory must be derived from empirical data. Research is a process of systematically collecting and analyzing data about a phenomenon through discovering, developing, and testing theories from the data. In this regard, research results are the theoretical presentation of reality (Glaser \& Strauss, 1968). Grounded theory has been widely used in factor research (Zakaria et al., 2016), for instance, identifying value-based factors in software development, while da Costa and Leite (2019) illustrate factors influencing research data communication on the Zika virus. Figure 1 shows the grounded theory research process.

This study draws on relevant literature and uses grounded theory for cause extraction and categorization. Following the basic steps of the grounded theory method, open coding is used to select representative sentences, form the risk factor, and attribute the key cause. Then, the basic factor categories are summarized and the potential structure relationships among the various categories is finally established. Grounded theory goes a long way in refining theoretical research and can effectively reduce the complexity of research topics. It also presents the current plurality of research topics and helps shape the extraction of major ongoing topic ideas. For these reasons, this method is used as the primary research method.

\subsection{Data source}

1. Research literature
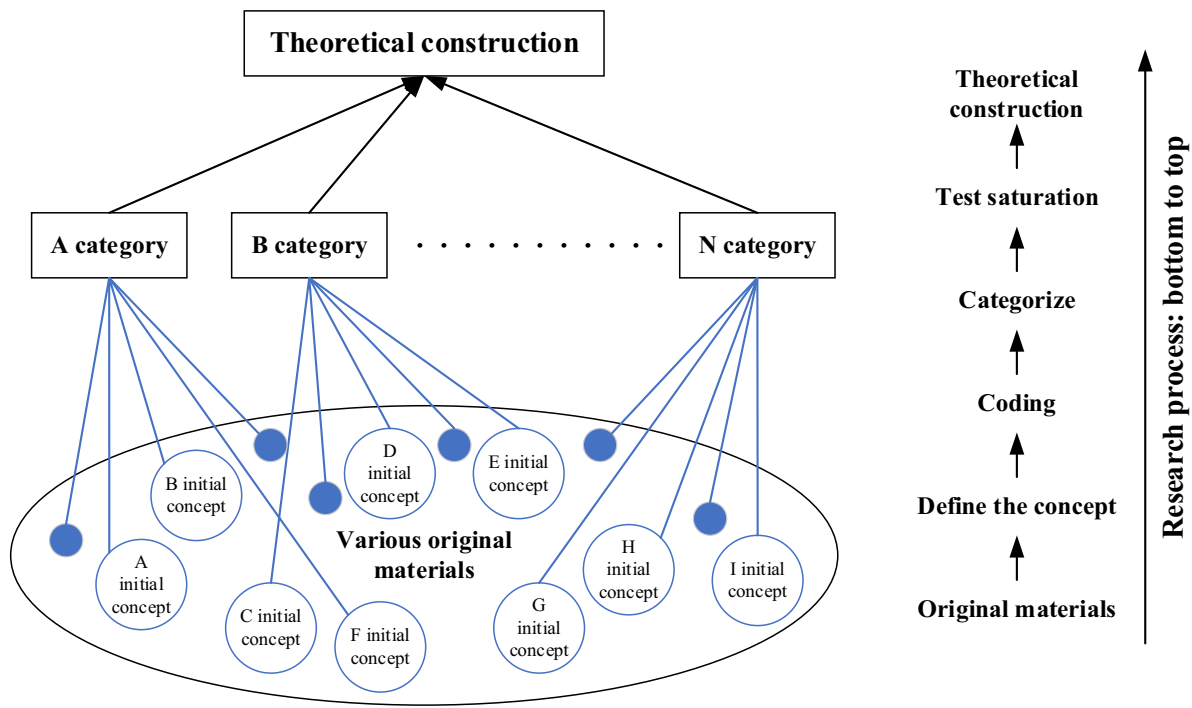

Fig. 1 The grounded theory research process 
To ensure the integrity and objectivity of the search results, the theme (multiple combinations of "city/urban", "innovative development", "innovative", "innovative system", "innovative ability", and "intelligent") was first adopted as the search expression. The search time span is "2000-2020", with the Web of Science database as the leading database and Elsevier and Scopus as supplementary databases. The preliminary searches obtained 776 initial records. At this point, the document abstracts were carefully reviewed, with documents obviously unrelated to urban innovation and development being deleted (i.e. those focusing on agricultural development, urban archives management, etc.). Documents such as "editions", "book reviews", and "newspaper articles" were not considered. Finally, documents covering duplicate topics in the literature were deleted. This resulted in a total of 45 articles. But, in order to ensure the retrieval literature was more representative, we complemented it with other documents from highly cited journals. Finally, a total of 60 articles were reviewed (Fig. 2). Table 1 lists the most influential journals in urban development research with the volume of total citations, g-index, h-index, and articles published from 2000 to 2020 . In this study, 60 literatures were selected from the articles published by these journals that are closely related to the influencing factors of urban innovation and development.

2. News reports

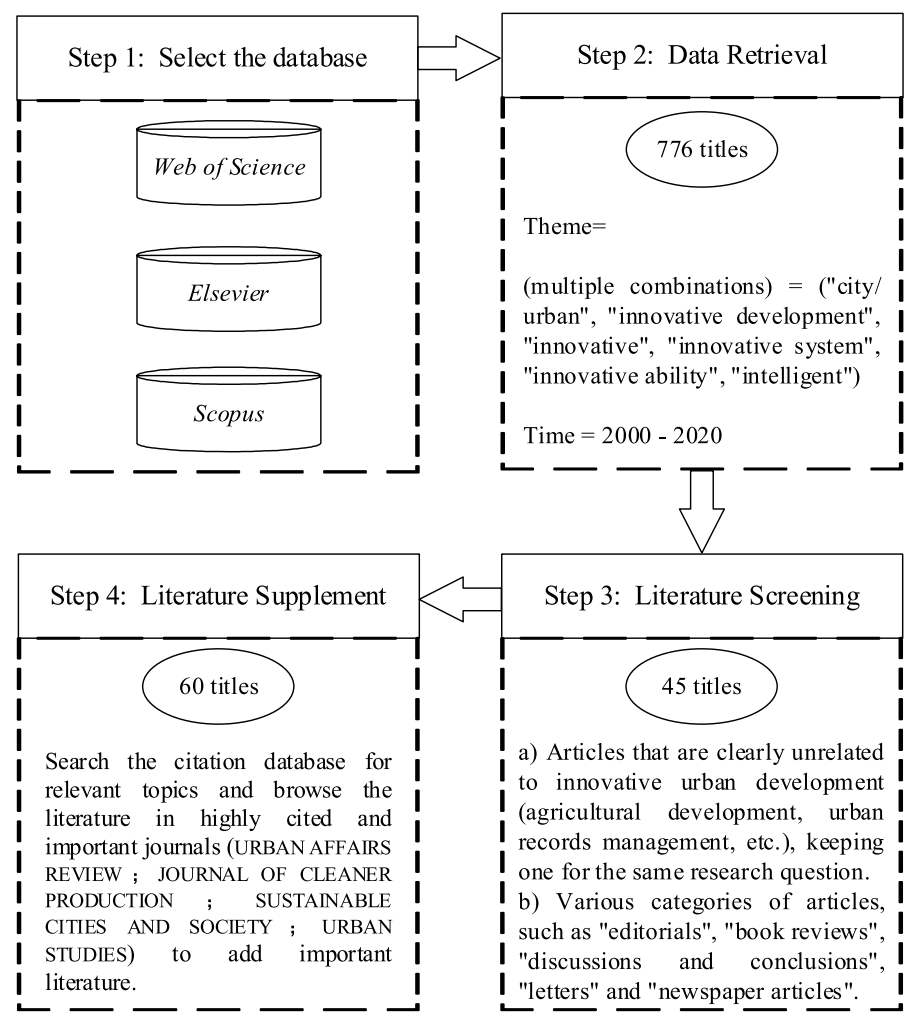

Fig. 2 Data collection process 


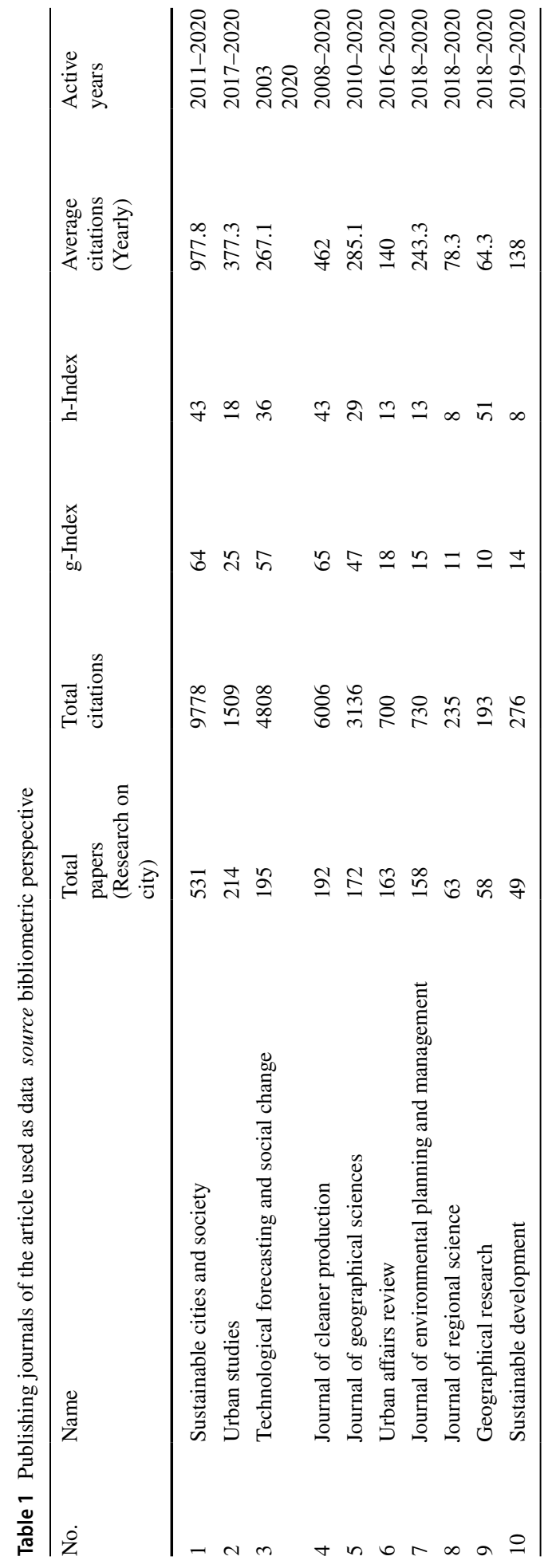


We searched in Google and Bing for keywords such as "urban/city innovation", "urban/ city innovation and development", "urban transformation", "innovative city", "smart city" and "urban reform", then reviewed the hits related to the subject. Finally, we selected 18 news reports published on the official website of the Central People's Government of the people's Republic of China (http://www.gov.cn), China city network (http://www.zgcsb. com), the United States Department of housing and urban development (https://www.hud. gov/), and the United Nations Development Programme (https://www.undp.org/).

\section{Interview data}

This study selected interview samples from the perspective of stakeholders, including urban residents, professionals, competent officials from decision-making departments, and experts. The distribution of research samples is shown in Table 2. Data were collected by face-to-face and telephone interviews. Some open questions about urban innovation and development were designed in advance, and then the interview questions focused on triggering a detailed discussion on the influencing factors of urban innovation and development. During this period, respondents could express their ideas without reservation, which has great advantages for the grounded theory three-level coding which requires a large number of texts and materials. A total of 23 people were interviewed in this study (15 men and 8 women, all aged 30-55, all with a bachelor degree or above). The total interview time of this study was 568 min. 43,855 words of written materials were collected.

\section{Data analysis process}

\subsection{Open coding: initial concept refinement}

Open coding mainly encodes the original data word by word. It gradually conceptualizes and categorizes the documents, and promotes the formation of more concepts and categories through constant comparisons. According to the requirements of open coding, 50 articles, 12 news reports and 18 interviews were firstly and randomly chosen for coding. Through labeling and repeated analysis of each document, several categories and initial

Table 2 Distribution of research samples

\begin{tabular}{lllr}
\hline No. & Sample type & Specific classification & Number \\
\hline 1 & Urban residents & Live in the city within ten years & 4 \\
& & Live in the city for more than ten years & 3 \\
2 & Professionals & Industrial enterprise & 2 \\
& & Construction enterprise & 4 \\
3 & Competent officials from & Municipal government & 2 \\
& decision-making depart- & Housing and urban rural development department & 2 \\
& ments & Expert in regional development research & 3 \\
& Experts & Experts in public policy & 3 \\
& & & 23 \\
\hline
\end{tabular}


concepts were finally abstracted from the text data (the same initial concept was not coded repeatedly). This process generated 37 initial concepts whose results are shown in Table 3.

\subsection{Spindle coding: determination of the main category}

The second major stage of grounded theory is spindle coding. This is responsible for the cluster analysis of the influencing factor codes obtained in the first stage. This analysis also identifies the possible logical and hierarchical relations of the influencing factor codes. Finally, it also classifies and establishes the relationships to form a more theoretical and instructive spindle code.

Hence, spindle coding carried out a clustering of the 37 initial concepts previously extracted and merged them into 12 main categories. These categories are shown in Table 4 and can be considered the major groups of influencing factors of 'urban innovation and development'.

\subsection{Selective coding: determination of core categories}

The third stage is selective coding, which systematically analyzes the relationship between categories from spindle coding. Compared to other categories, the core category should have a commanding nature and be able to encompass most research results in a relatively broad range. From the two previous coding, we conducted repeated analyses and comparisons of the relationships between the main categories and abstracted the 12 main categories into three core categories. The three major groups of factors affecting urban innovation and development were social factors, economic factors, and natural factors. They are summarized in Table 5.

\subsection{Saturation test}

The saturation test in this case consisted of reserving 10 articles, 6 news reports and 5 interviews to test whether the current influencing factors were sufficiently comprehensive. Using the reserved literature, coding was continued using the NVivo11 software tool, which enabled extraction of the influencing factors that fully coincided with the existing coding concepts. Therefore, it can be concluded that the influencing factor coding is sufficiently rich as no new urban innovation and development impact factors were found. Consequently, it was determined that the proposed influencing factors list had reached saturation point.

\section{Influencing factors of urban innovation and development}

\subsection{Social factors}

Social factors are the influencing factors included in the social system of urban innovation and development. The social system is restricted by population, policy, and social structure. Culture, scientific level, and traditional habits are all factors that must be considered when analyzing the relationship between social organizations and human activities. These factors are closely related to the innovation and development of cities and urban centers 


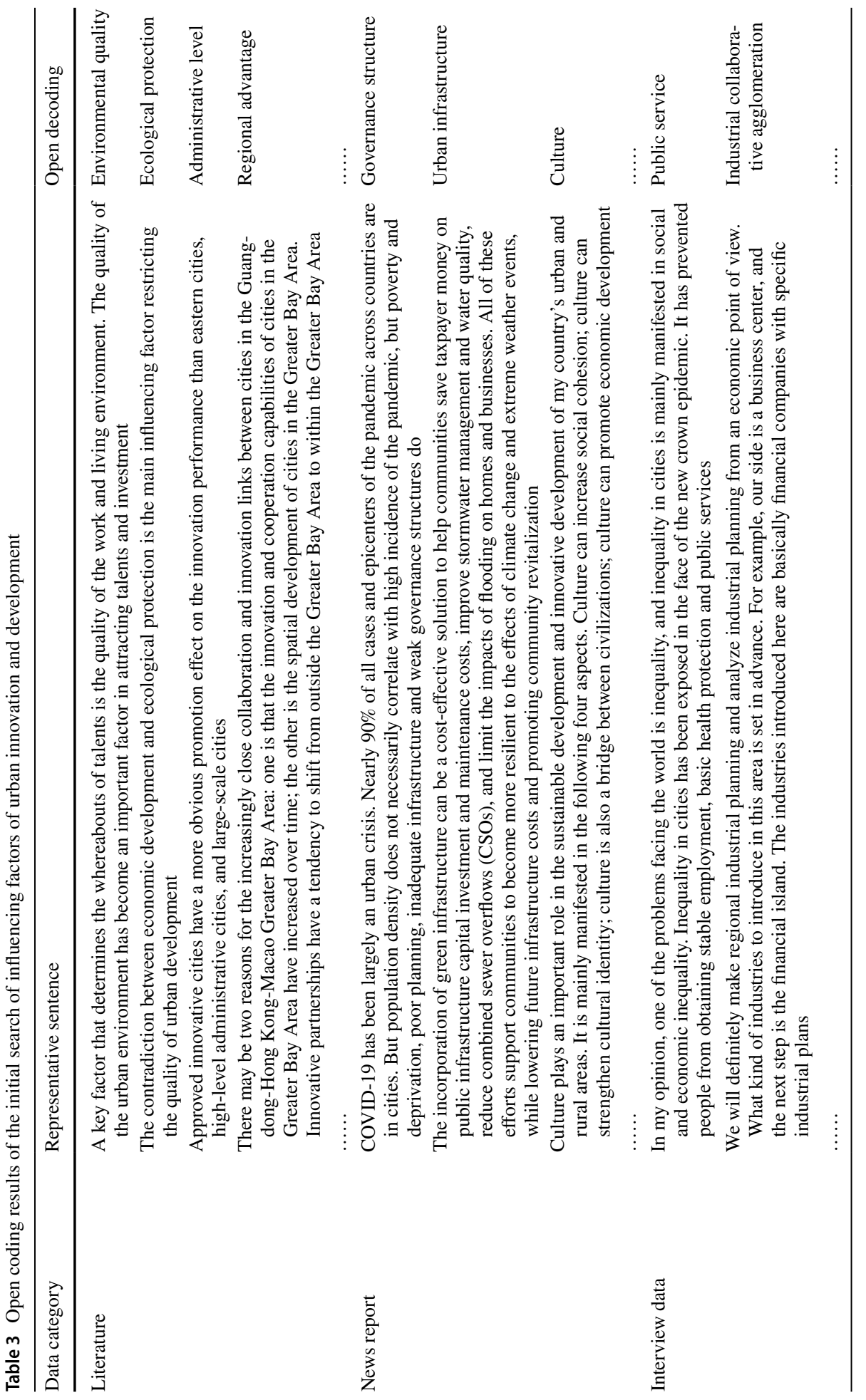


(Ma, 1984; Tan et al., 2008; Barrado-Timón et al., 2020). The qualitative analysis results identify the social factors that affect the innovation and development of cities to be cultural environment, administrative levels, government management, service measures, and population structure (see Fig. 3).

\section{Administrative level}

The different administrative levels of cities are similar to geographical locations, thereby representing different resources. Indeed, most cities with a higher administrative level have convenient forms of transportation, sound infrastructure, and higher levels of physical openness. These advantages have promoted a number of other factors such as accumulation (Cartier \& Carolyn, 2011; Wei, 2015), which, in turn, promote urban innovation and development. At the same time, in the process of promoting the development of local governments, the central government also tends to allocate more resources to the capital, municipalities directly managed by the central government, and cities specifically designated in the regional or national governmental plan. In this context, provinces and autonomous regions tend to favor province capitals as well as capital cities because they are the preferred locations for capital investments and other economic and social support mechanisms (Cainelli et al., 2021; Peng et al., 2016).

\section{Cultural environment}

Culture has an important strategic position for the development of a city, with the cultural environment having a supporting and leading role in innovation and development (Lazzeroni et al., 2013). Moreover, cities with rich cultural assets and cultural industries are more likely to stimulate the vitality of innovative entities and generate new ideas leading to greater innovation capacity. For example, in China, Shenzhen's innovation and development has benefited from its rich, open, inclusive, and diverse entrepreneurial culture, which has created a supportive and enabling innovation environment (Gu et al., 2016). At the same time, interviews with enterprise personnel also indicated that the innovation model with enterprises as the main body and integrating production, education and research can attract all kinds of innovative and entrepreneurial talents, form an atmosphere of innovation, and stimulate the vitality of urban development.

\section{Population structure}

The coordination strategy of supporting education levels as well as forms of diversity in populations can have a great influence on the city's innovation capability (Huang et al., 2014; Luca \& Margherita, 2016). Indeed, a city population with a high density of educated inhabitants is more conducive to promoting regional economic growth (Glaeser, 1998; Armeanu et al., 2017). Therefore, the population education structure has a significant impact on urban innovation and development. Concentrating high-quality talents, frequent contacts, and exchanges with each other further stimulates the spirit of innovation, which is undoubtedly conducive to the generation of new ideas as well as the development of new technologies. In the process of interviews, government personnel also emphasized the importance of urban population structure and cultural quality. That is, a reasonable population structure can effectively control unemployment, reduce urban social instability, and promote urban social development. 


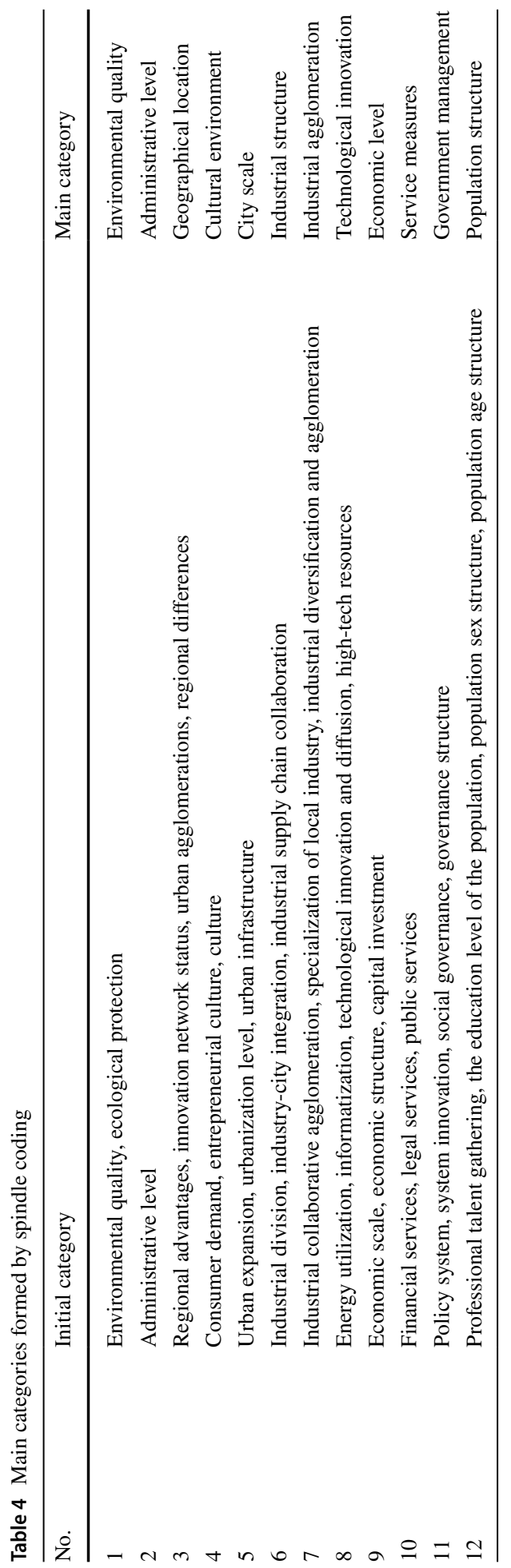


Table 5 Core category formed by selective coding

\begin{tabular}{lll}
\hline No. & Main category & Core category \\
\hline 1 & Cultural environment & Social factors \\
& Administrative level & \\
& Government management & \\
& Service measures & \\
& Population structure & \\
& Economic level & Economic factors \\
& Industrial structure & \\
& Industrial agglomeration & \\
& Technological innovation & Natural factors \\
& Ecological environment & \\
& Geographical location & \\
& City scale &
\end{tabular}

\section{Government management}

Urban innovation and development are a complex and dynamic process that requires long-term planning rather than short-term interventions. This process relies on the provision and coordination of supporting mechanisms and appropriate regulations and controls by governmental entities. The government's coordination and promotion role provides policy and a supportive environment for urban innovation and development. For example, in the early stages of the development of high-tech industries, there can be obvious market failures where government intervention and management becomes beneficial (MA et al., 2013; Neves \& Branco, 2020). Cities with high quality social governance often have a higher quality of innovation and development (Liu et al., 2017). The report "Innovative Ideas to Promote the Modernization of Urban Governance System and Governance Capability" also pointed out the need to promote the innovation of

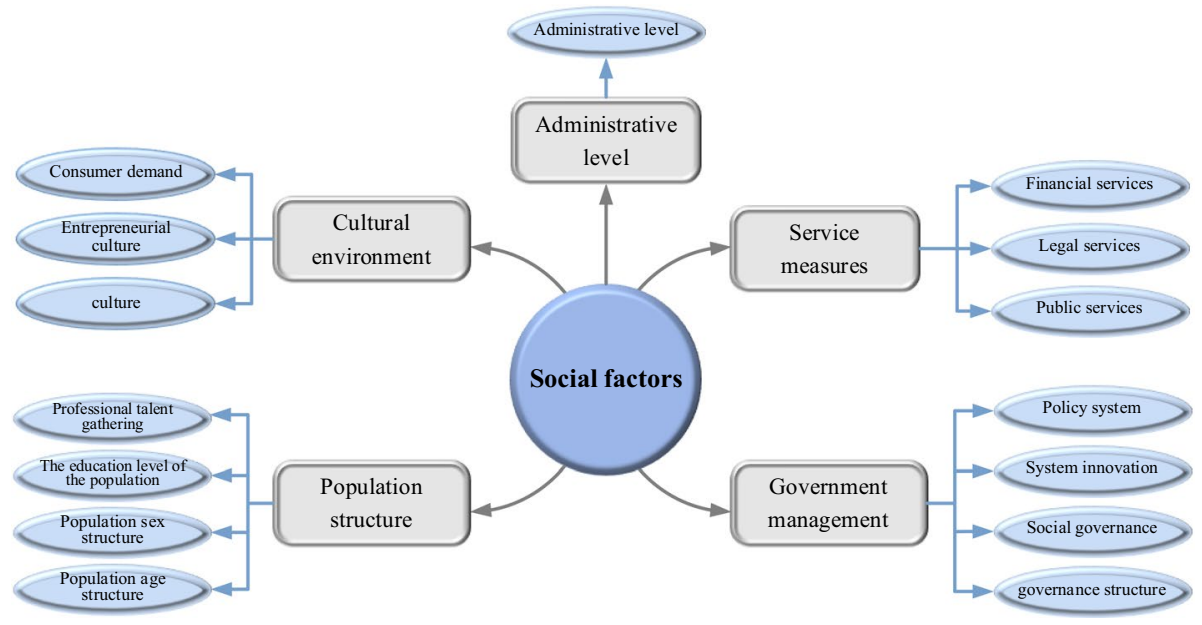

Fig. 3 Social factors affecting urban innovation and development 
urban management methods, management models, and management concepts to make cities become smarter.

\section{Service measures}

Service measures include not only public services with the government as the main body, such as transportation planning, convenient infrastructure and perfect system guarantee, but also enterprises' service behaviors aimed at consumers. From a macro perspective, urban service innovation directly affects the image and charm of the city (Gammal \& Abdelsabour, 2016). For citizens and organizations residing or working in the locality, their functioning makes the normal course of business of the whole city (Buhociu et al., 2011). In addition, effective legal and financial services are also important factors that attract the accumulation of innovative elements (Rybinski, 2016).

\subsection{Natural factors}

Although natural science was originally a discipline dealing with the relationship between plants and animals and their habitats or environment, it has also been applied in the fields of physiology, mathematics, and economics, as well as urban planning (Lenicki \& Lewandowska, 2016, Wang et al., 2019). The applied research dimension of natural sciences in urban development focuses on urban ecology, which has introduced the ecological viewpoints of plants and animals into human communities. Related concepts such as "environment", "natural process", "competition", and "symbiosis" can be used to analyze cities or communities. According to the qualitative analysis results, the natural factors affecting the innovation and development of cities mainly include ecological environment, city scale, and geographic location (see Fig. 4).

\section{Ecological environment}

The city can be viewed as a "society-economy-natural complex ecosystem", and the harmonious coexistence of humans and nature is a key component of urban innovation and development (Ma, 1984). Based on the concept of sustainable development, high-tech industries in the twenty-first Century generally have very high requirements for the ecological environment. This can result in excessive emissions (e.g. carbon dioxide and particles) and the resulting pollution. Once the environment is negatively impacted and eventually destroyed, the situation can lead to a loss of human resources and high-tech industries, which further damages the city's future. In contrast, an effective environmental protection policy can be an important enabler to attract human resources and capital investment to an urban center (Khan et al., 2020). In addition, the report issued by the U.S. Department of Housing and Urban Development shows that renewable energy has very real economic and ecological benefits, encouraging clean jobs for our communities and driving the economy. In this regard, the contradiction between economic development and ecological protection is the main influencing factor restricting the quality of urban development (Gao \& Mei, 2013; Kopnina et al., 2020).

\section{City scale}


In essence, the scale of a city also reflects the abundance of urban resources. Largescale cities often have readily available capital for investment purposes and diversified resources as well as diversified cultures, all of which can contribute to the development of innovative activities in urban centers (Packalen \& Bhattacharya, 2015). Furthermore, large-scale cities benefit from agglomeration effects resulting from economies of scale and network effects. As explained by urban residents in interviews, more and more young people choose to move to big cities. This as big cities can provide more opportunities, and they are accustomed to their convenience provided by big cities. These can also lead to greater economic activity, which, in turn, attracts further capital investment and results in a further attraction of human resources to the urban center in a virtuous circle of urban innovation and development.

\section{Geographical location}

The difference in urban geographic locations involves differences in traffic conditions, the level of opening to the outside world, and the concentration of human resources, as well as central government policies (Neirotti et al., 2014; Hutzschenreuter \& Harhoff, 2020). In the case of China, the innovation efficiency of Chinese cities has a decreasing trend from southeast to west (Du et al., 2019)-the innovation efficiency of cities in the eastern region being significantly higher than the central and western regions. This is determined by the advantages of the cities' own resource endowments in the eastern region. There are also large regional differences in the speed of improvement. The triangle of central China and the southwest region have the fastest growth rates, which are much higher than the coastal regions and the northeast region (Zhou et al., 2019).

\subsection{Economic factors}

Economic activity is associated with a series of activities that people pursue to organize production, circulation, and consumption for their own survival and development. Materials and energy are collected, processed, and produced in economic activities to become

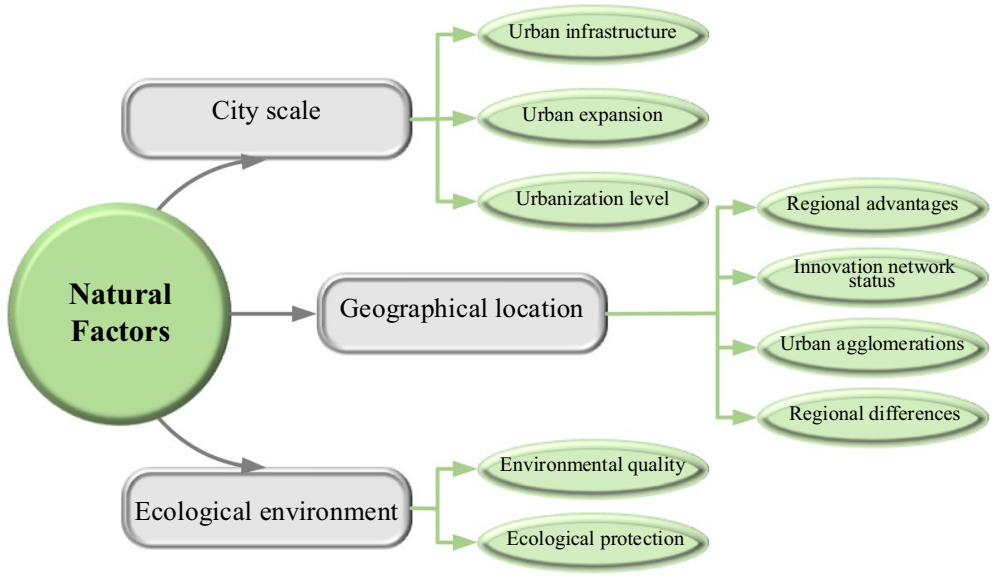

Fig. 4 Natural factors affecting urban innovation and development 
economic products, some of which become consumer goods, and some of which are used to expand production. In this respect, people and societies at large turn natural materials and energy into products they need to meet immediate and long-term development needs. Thereby, industries are formed (Yao et al., 2015). The essence of the internal interaction of the complex system is the flow and transformation of material. Therefore, when considering economic factors in the process of urban innovation and development, this study mainly analyzes the material form of economic input and output, rather than their monetary form. Based on the qualitative analysis results, the economic factors affecting the development of urban innovation mainly comprise economic level, technological innovation, industrial agglomeration, and industrial structure (see Fig. 5).

\section{Economic level}

Economic level is mainly reflected in the city's economic structure and economic scale. Economic structure is, in fact, a complex and dynamically adaptable organizational process where its lock-in will lead to the decline of the city. In the process of urban development, lock-in occurs because the existing economic structure has severely hindered the generation of urban innovation capabilities, resulting in cities not adapting to the economic change process (Perkins, 2012; Zhang et al., 2020). Economic scale can be viewed as the soil from which innovation grows. It is necessary to first create a supportive environment for the development of enterprises to improve the city's innovation capability. A city with strong economic strength can provide enterprises with effective infrastructure as well as enabling infrastructure in terms of government procurement, tax incentives, and financial assistance, thereby creating more favorable conditions for enterprises to develop and become financially sustainable (Wen, 2011; Accetturo et al., 2019).

\section{Industrial agglomeration}

Industrial agglomeration will inevitably be accompanied by the agglomeration of innovation elements, such as innovative human resources and innovation capital. This is conducive to the innovation capital reservoir effect and the human capital reservoir effect. It also promotes the improvement of the city's innovation level (Chen et al., 2013). Industrial agglomeration strengthens the cooperation between the industrial supply chain upstream and downstream ends, maximizes knowledge and technology exchange, promotes knowledge spillover, and promotes industrial development. The industrial agglomeration mechanism also results in different effects on urban development due to the difference in city location. In the case of China (Liu \& Zhang, 2017), for the eastern region, both specialized agglomeration and diversified agglomeration help improve the innovation capabilities of cities in the region. Whereas, for the central and western regions, professional agglomeration significantly improves the innovation capabilities of the cities there. Both mechanisms can form a knowledge and technological environment conducive to R\&D in the agglomeration area, promote the speedier transfer and diffusion of knowledge and technology, and form strong competition between enterprises. This ultimately encourages enterprises to improve their innovation level and the overall innovation level of industrial clusters. 
3. Industrial structure

An effective industrial structure can improve the quality and efficiency of technological innovation, thereby promoting urban innovation and development. The process of industrial restructuring is also a process of re-allocating innovative resources, which is an industry with a higher innovation level. In this process, innovation elements flow from lower to higher innovation industries. This is conducive to the rational allocation of innovation factor resources and improves the level of urban innovation (Yang et al., 2019). In the interview, government officials also pointed out that they will develop a regional industrial planning. What kind of industries will be introduced in the city should be set in advance, and the innovation chain will be deployed around the industry chain. In turn, the industry chain will be laid out around the innovation chain. There are two main ways to adjust the urban industrial structure: one is by relying on technological innovation to transform traditional industries; another is by introducing emerging industries and high-end service industries to replace traditional industries. The biggest gap in urban industrial restructuring is often technological. Therefore, industrial restructuring forces cities to develop technological innovation (Li \& Yang, 2017).

\section{Technological innovation}

In the process of transforming the industrial economy to the knowledge economy of the twenty-first Century, scientific and technological innovation are an inevitable pathway to improve the competitiveness of urban centers. Technological innovation promotes technological progress, thereby improving the urban industrial and employment structure. For example, the development of informatization can enhance the integration of city level functions and the competitiveness of cities through informatization. These are successful experiences observed in many countries and regions (Zhou et al., 2020). Zhang et al. also shows that technological innovation can affect the development of urban innovation, and the impact on eastern cities is greater than that on central and western cities. This impact is also related to the administrative level of the city (Zhang et al., 2018). The impact of technological innovation on urban innovation and development is realized by acting on industrial development. Technological innovation drives the upgrading of industrial structure and optimization of physical layout, improves energy efficiency, and promotes the greening

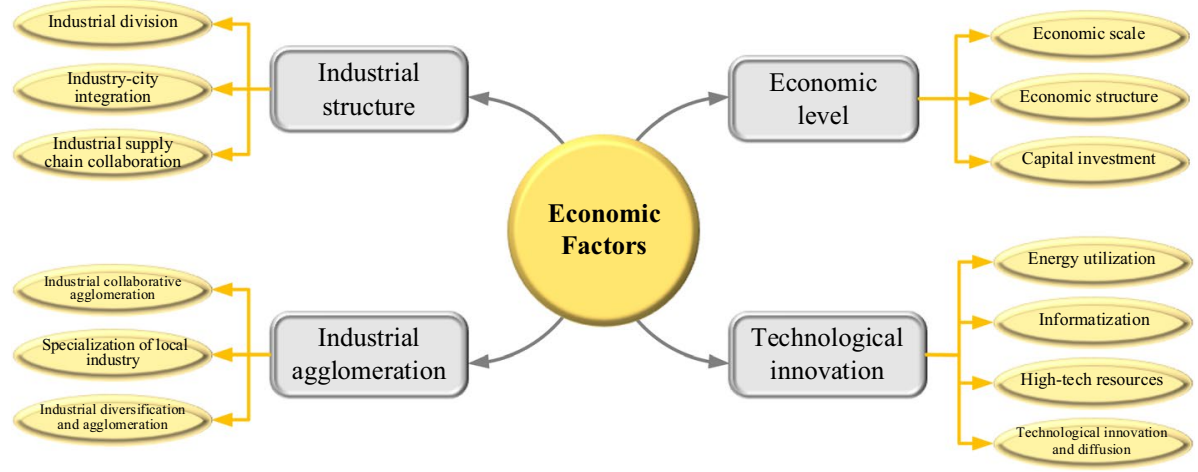

Fig. 5 Economic factors affecting urban innovation and development 
of urban economy, together with the efficient and intensive use of urban space, energy, and resources. All this enables the innovative development of cities (Chen et al., 2021; Yuan \& Zhang, 2020).

\subsection{Interaction between the influencing factors}

Urban innovation and development involve interactions between multiple participants and multiple levels and does not necessarily follow traditional models: this means that cities need to implement new or existing activities in new ways in the innovation process. As a micro-ecosystem, the city maintains material and energy exchanges with the external environment during the development process. Therefore, when considering the innovative development of the city, we have identified that it needs to be related to the necessary natural, social, and economic factors. Indeed, natural factors, social factors, and economic factors occupy different positions in the process of urban innovation and development by interactively and coordinately influencing urban innovation and development from different sides. Based on the results of the qualitative analysis, Natural factors are the sum of all conditions in the process of generation, operation, and effect, and need to be considered first in the innovation and development of cities. Social factors indicate the inherent limitations and conditions of urban innovation and development. Economic factors are to a large extent influencing the decisions of policy makers, and ultimately determining the direction and extent of urban innovation and development. Therefore, urban innovation and development is within the large range of natural factors, within the specific limits of social factors, and embodied in economic factors. Accordingly, an onion model of factors affecting urban innovation and development is proposed (see Fig. 6).

Natural factors are prerequisite for urban innovation and development, which needs to harness resource endowments and adjust measures to the local conditions of the urban center. To improve industrial development, cities lacking funds must abandon the catch-up in capital-intensive industries. The development of industries also affects the city's natural environment. For example, industrial upgrading promotes the city's expansion. Moreover, innovation not only affects its own development, but also that of other cities: the activity establishes connections with surrounding cities, directly or indirectly affecting the local city's status in the regional innovation network.

Economic factors are the key factors for urban innovation and development. Urban development is based on industrial activity and growth and constitutes a complex internal interaction model. Moreover, industrial development and urban development are inseparable: the industrial system and the urban system penetrate and self-regulate each other, mutually promoting the spiral development model and the development pattern of "industry-city double-strength". The separation of industry and city enables innovation activities to be not only effectively supported by urban functions, but also without strong support for industrial development (Wang et al., 2020).

Social factors are the guarantee factors for urban innovation and development: urban development requires the protection of the social system. There is a need for the necessary capital investment, a reasonable population structure, gathering of high-tech human resources, guidance and coordination of government policies, a sound legal guarantee mechanism, and financial services. All this will promote industrial upgrading and thus urban innovation and development. The social system provides a guarantee for industrial development and needs to adapt to its development according to the different stages and modes of industrial development. At the same time, the advancement of science and 
technology also requires the management and service methods of the social system to be upgraded. All this will facilitate the healthy and steady improvement of the city's economic level.

Natural factors, economic factors, and social factors also have mutual influences and interdependencies. The impact of natural factors on social factors is mainly due to the accumulation of human resources caused by large-scale cities, which increases the education level of the population. Government policies are inclined toward cities with high administrative levels. In the case of China, cities in the eastern region have accumulated a large number of innovative elements and have advantages in terms of economic level and services (Du et al., 2019). Natural factors are counteracted by the performance of such social factors as the guidance of government policies making the city's environmental protection atmosphere stronger, social governance and services improving the ecological appearance of the city, and increasing the level of economic development expanding the city's scale. Economic factors, as key factors in urban innovation and development, are based on natural factors and are protected by social factors: these, in turn, have a direct impact on the development of urban innovation.

\section{Conclusions and recommendations}

Based on grounded theory, this study used NVivo11 software to code and analyze literatures, news reports and interview materials relating to urban innovation and development published. The following results are obtained. First, the influencing factors of the urban innovation and development of cities are "environmental quality", "geographical location", and "city scale" from a natural perspective; "economic level", "industrial structure", "industrial agglomeration", and "technological innovation" from an economic perspective; and "administrative hierarchy", "cultural environment", "population structure", "government management", and "service measures" from a social perspective. Second, natural factors are prerequisites (or enabling factors), and therefore need to be considered first in the process of urban development. Economic factors are the main factors involved. Namely,

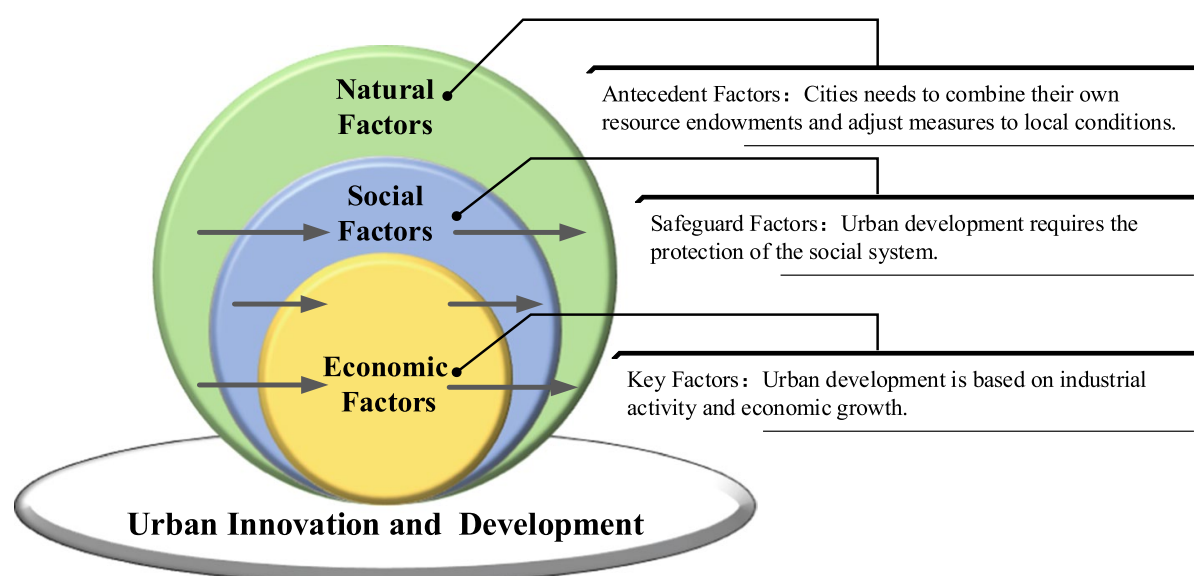

Fig. 6 Onion model of factors affecting urban innovation and development 
a high-quality development of cities is a reflection of the healthy and stable development of economic activities in the urban center. Social factors are the guarantee factors for the innovation and development of cities. This means they have a supporting effect as part of this process. The impact of the three major groups of factors on the development of urban innovation is not isolated as they have significant interrelationships.

Based on the results above, three suggestions are proposed for promoting the innovative development of cities.

(1) Natural factors, as the prerequisites for the innovative development of cities, should be the primary concern for policy makers. Urban innovation needs to match geographical location with the available resources to support innovation and the environmental conditions of the region. Innovative development strategies should be adapted to local conditions and not blindly assume that one size will fit all situations. Cities with a richer set of innovation-related resources (such as higher education institutions and a highly trained workforce together with access to capital for investment) may need to adopt a different development strategy. For instance, the primary strategy for urban centers with weaker resources may be to attract more highly skilled and/or educated workers, then upgrade the industrial infrastructure of the city.

(2) The carrier of economic activity at the city level is industry, and industrial development underpins the process of urban development. Urban innovation and development are inseparable from the upgrading and development of industries. Therefore, the government needs to consider the coordination of industrial activities and urban development from a long-term perspective. This may be achieved by providing a supportive industrial foundation for urban innovation and development, and by actively promoting the development of industrial agglomeration. The latter enables economies of scale, innovation, and competition effects of industrial agglomeration. It is suggested that urban centers strengthen the level of technological innovation of their industrial sector together with encouraging interactions with higher education institutions as part of university-industry research collaborations and technology transfer activities.

(3) Third, urban innovation and development should include a necessary focus on the social environment. Cities need to create a supportive cultural and social environment for attracting high-tech industries and highly skilled workforce. In the process of development, they should reduce the barriers to talent introduction. This means they need to improve the incentives system and increase the city's cultural foundation to create a diverse and tolerant environment that maintains its long-term and stable attractiveness to talent and industries. In addition, the government's financial support and healthy financial environments play a vital role in industrial development. The government needs to ensure the necessary services are present to support and improve the market mechanism to enhance the rational level of factor flow and capital allocation.

\section{Limitations and further research}

Due to the characteristics of grounded theory, this study only establishes a more general model of the interaction of influencing factors in the innovation and development of cities. This lays the foundation for further research. Additionally, huge differences between cities (at the administrative level, and/or geographical location, for example) mean that this 
theoretical model still has room for further specialization. Therefore, future research might benefit from our proposed theoretical system, to carry out more detailed research on specific cities and enrich this first model with a combination of qualitative and quantitative research methods.

Funding This research is supported by the National Social Science Fund projects (No.20BJY010);National Social Science Fund Post-financing projects (No.19FJYB017); Sichuan-tibet Railway Major Fundamental Science Problems Special Fund (No.71942006); List of Key Science and Technology Projects in China's Transportation Industry in 2018-International Science and Technology Cooperation Project (No.2018GH-006 and No.2019-MS5-100); Emerging Engineering Education Research and Practice Project of Ministry of Education of China (No.E-GKRWJC20202914); Shaanxi Social Science Fund (No.2017S004); Xi'an Construction Science and Technology Planning Project (No.SZJJ201915 and No.SZJJ201916); Shaanxi Province Higher Education Teaching Reform Project (No.19BZ016); Fundamental Research for Funds for the Central Universities (Humanities and Social Sciences), Chang'an University (No.300102230612).

\section{References}

Accetturo, A., Lamorgese, A., Mocetti, S., et al. (2019). Local development, urban economies and aggregate growth. Italian Economic Journal: A Continuation of Rivista Italiana Degli Economisti and Giornale Degli Economisti [J], 5, 191-204.

Alhaddi, H. (2015). Triple bottom line and sustainability: A literature review. Business and Management Studies [J], 1, 6-10.

Ari-Veikko, A. (2016). City-as-a-platform: The rise of participatory innovation platforms in finnish cities. Sustainability [J], 8, 922.

Armeanu, D., Vintilă, G., \& Gherghina, Ş. (2017). Empirical study towards the drivers of sustainable economic growth in EU-28 countries. Sustainability [J], 10, 4.

Barrado-Timón, D., Palacios, A., \& Hidalgo-Giralt, C. (2020). Medium and small cities, culture and the economy of culture. A review of the approach to the case of Spain in light of international scientific scholarship. Sustainability [J], 12, 7321.

Buhociu, F. M., Mihalcea, L., \& Virlanuta, F. O. (2011). Economic evaluation of a public service potential of a municipality-urban development in Romania. Risk in Contemporary Economy [J], 1, 12-17.

Cainelli G, Ciccarelli C, Ganau R. (2021). Administrative reforms, urban hierarchy, and local population growth. Lessons from Italian unification. Papers in Evolutionary Economic Geography (PEEG) [J].

Cartier, C. (2011). Urban growth, rescaling, and the spatial administrative hierarchy. Provincial China [J]. https://doi.org/10.5130/pc.v3i1.2440

Chen, J., Liang, L., \& Hang, W. U. (2013). Industrial agglomeration and innovation performance under the background of open innovation: evidence from Chinese high-tech industries. Studies in Science of Science $[J], 4,623$.

Chen, M., Sinha, A., Hu, K., et al. (2021). Impact of technological innovation on energy efficiency in industry 4.0 era: Moderation of shadow economy in sustainable development. Technological Forecasting and Social Change [J], 164, 120521.

Chen, L., \& Xu, X. N. (2009). Construction and operational mechanism of innovative city's innovation system. Pioneering with Science \& Technology Monthly [J], (5), 3.

Coscieme, L., Mortensen, L. F., \& Donohue, I. (2021). Enhance environmental policy coherence to meet the sustainable development goals. Journal of Cleaner Production [J], 296, 126502.

da Costa, M. P., \& Leite, F. C. L. (2019). Factors influencing research data communication on Zika virus: A grounded theory. Journal of Documentation [J], 75, 910-926.

Deng Z, Chen Y. (2020). Research on place-making in innovation districts. City Planning Review [J].

Dong, J. L. (2014). Study on innovation factors of industrial transformation in the old industrial city. Advanced Materials Research [J], 962-965, 2529-2532.

Du, J. L., Liu, Y., \& Diao, W. X. (2019). Assessing regional differences in green innovation efficiency of industrial enterprises in China. International Journal of Environmental Research and Public Health $[\mathrm{J}], 16,940$.

Elkington, J. (1998). Partnerships from cannibals with forks: The triple bottom line of 21st-century business. Environmental Quality Management [J], 8, 37-51. 
Eskov, V. M., Eskov, V. V., Filatova, O. E., et al. (2012). Two types of systems and three types of paradigms in systems philosophy and system science. Journal of Biomedical Science and Engineering [J], 5, 602-607.

Gammal, M. A., Abdelsabour, E. (2016). Technology era city models and governance urban innovation integrated planning. SBE 16 Cairo.

Gao, M. H. (2013). Thinking of economic development policies based on ecological environment protection. Advanced Materials Research [J], 726-731, 4185-4189.

Glaeser, E. L. (1998). Are cities dying? Journal of Economic Perspectives [J], 12, 139-160.

Glaser, B., \& Strauss, A. L. (1968). The discovery of grounded theory: Strategies for qualitative research. Nursing Research, 17, 377-380.

Gu, S., Mei, Y., \& Zhuang, Q. (2016). Strategic thinking on developing innovative city to implement the strategy of innovation-driven development_— based on the model of Shenzhen innovative development. Forum on Science and Technology in China [J], (9), 7.

He, Y. Q., Pan, X. X., Wang, Y., et al. (2013). Evaluation and analysis on coupling coordinated development of urban resource, environment and economy in Jiangxi province in China. Applied Mechanics and Materials [J], 295-298, 2457-2463.

Hou R, Zhang L, Wang A. P., et al. (2009). Regional innovation ability evaluation index system and its empirical research. Journal of Wuhan University of Technology (Information \& Management Engineering) $[\mathrm{J}]$.

Huang, R., Liang, Q., \& Lachang, L. V. (2014). Relationship of urban population structure and innovation ability: A case study of Urban China. Urban Development Studies [J], 21, 84-91.

Hutzschenreuter, T., \& Harhoff, P. L. (2020). National capital city location and subsidiary portfolio expansion: The negative effect of geographic distance to the capital city at inception on the speed of subsequent investments. Journal of International Business Studies [J], 51, 1107-1132.

Jagt, A., Raven, R., Dorst, H., et al. (2020). Nature-based innovation systems. Environmental Innovation and Societal Transitions [J], 35, 202-216.

Khan, I., Hou, F., \& Le, H. P. (2020). The impact of natural resources, energy consumption, and population growth on environmental quality: Fresh evidence from the United States of America. Science of the Total Environment [J], 754, 142222.

Kopnina, H., Washington, H., Lowe, I., et al. (2020). Scientists' warning to humanity: strategic thinking on economic development, population, poverty and ecological sustainability in the Mediterranean and beyond. Euro-Mediterranean Journal for Environmental Integration. https://doi.org/10.1007/ s41207-019-0139-4

Krishna Kumari, A. (January 10, 2007). Understanding Sustainable Development. Available at SSRN: https://doi.org/10.2139/ssrn.956240

Lauer, J., \& Liefner, I. (2019). State-led innovation at the city level: Policy measures to promote new energy vehicles in Shenzhen, China. Geographical Review [J], 109, 436-456.

Lazzeroni, M., Bellini, N., Cortesi, G., et al. (2013). The territorial approach to cultural economy: New opportunities for the development of small towns. European Planning Studies [J], 21, 452-472.

Lenicki, M., \& Lewandowska, A. (2016). Contemporary concepts of a city in the context of sustainable development: Perspective of humanities and natural sciences. Social Science Electronic Publishing $[J], 11,45-54$.

Li, H., Wu Y. (2015). The influence factor and the vision design of the "Human Urbanization": Observation of a municipality directly under the central government. Reform [J], (11), 10.

Li, Z., \& Yang, S. Y. (2017). Science and technology innovation, industrial upgrading and economic growth: Interactive mechanism and empirical test. Jilin University Journal Social Sciences Edition $[J] ., 57,41-204$.

Li, L., \& Zhang, X. (2020). Spatial evolution and critical factors of urban innovation: Evidence from Shanghai, China. Sustainability [J], 12, 938.

Liu, L., Zhong-Lei, Y. U., Xiao-Hong, X. U., et al. (2016). Coupling coordination degree of city innovation capability and urbanization in Shandong Province. Economic Geography [J], 36, 59-66.

Liu, P., Zhang Y. F. (2017). Industrial agglomeration, FDI and urban innovation capability-Spatial Durbin model based on the data of 264 prefecture-level cities in China. East China Economic Management $[\mathrm{J}], 031(005), 56-65$.

Liu, J. F., MANAGEMENT S. O., UNIVERSITY Q. N. (2017). From Social Management to Social Governance: Experience, Reflection and New Normal of Innovation-an Analysis of the 38 Comprehensive Pilot Areas of Social Management Innovation. Academic Forum of Nandu [J], 37 (4), 7.

Luca, S., \& Margherita, C. (2016). In-between stability and subtle changes: Urban growth, population structure, and the city life cycle in Rome. Population Space and Place [J], 22, 216-227. 
Ma, S. (1984). The social-economic-natural complex ecosystem. Acta Ecologica Sinica [J], 1, 2006.

Ma, H. T., Fang, C. L., Wang, S. J. (2013). Indicators of global innovative cities and their implications for China. Urban Planning Forum [J], (1), 9.

Markatou, M., \& Alexandrou, E. (2015). Urban system of innovation: Main agents and main factors of success. Procedia-Social and Behavioral Sciences [J], 195, 240-250.

Marzluff, J., Shulenberger, E., Endlicher, W., et al. (2008). Integrating humans into ecology: Opportunities and challenges for studying urban ecosystems. Urban Ecology [J]. https://doi.org/10.1007/ 978-0-387-73412-5

Neirotti, P., Marco, A. D., Cagliano, A. C., et al. (2014). Current trends in smart city initiatives: Some stylised facts. Cities [J], 38, 25-36.

Neves, E. D., \& Branco, J. R. (2020). Determinants of R\&D on European high technology industry: Panel data evidence. Paula Fernandes [J], 18, 285-305.

Nielsen, S. N. (2007). What has modern ecosystem theory to offer to cleaner production, industrial ecology and society? The views of an ecologist. Journal of Cleaner Production [j], 15, 1639-1653.

Njøs, R., \& Jakobsen, S.-E. (2016). Cluster policy and regional development: Scale, scope and renewal. Regional Studies. Regional Science [J], 3, 146-169.

Packalen, M., Bhattacharya, J. (2015). Cities and ideas [M]. National Bureau of Economic Research.

Peng, L. I., Liu, R. Y., \& Jian, L. I. (2016). Administrative hierarchy and resource allocation efficiency of Chinese city. Economic Geography [J], 36, 46-51.

Perkins, D. H. (2012). Rapid growth and changing economic structure: The expenditure side story and its implications for China. China Economic Review [J], 23, 501-511.

Pickett, S. T. A., Cadenasso, M. L., et al. (2001). Urban ecological systems: Linking terrestrial ecological, physical, and socioeconomic components of metropolitan areas. Annual Review of Ecology and Systematics [J], 32, 127-157.

Popescu, A. I. (2020). Long-term city innovation trajectories and quality of urban life. Sustainability, 12, 10587.

Rybinski, K. (2016). Innovation in financial services [C]: Innovation in emerging markets (pp. 196-214). London: Palgrave Macmillan.

Scalia, M., Angelini, A., Farioli, F., et al. (2020). An ecology and economy coupling model. A global stationary state model for a sustainable economy in the Hamiltonian formalism. Ecological Economics [J], 172, 106497.

Schumpeter, J. A. (1947). Theoretical problems of economic growth. Journal of Economic History [J], 7, 1-9.

Simmie, J., \& Sennett, J. (1999). Innovative clusters: Global or local linkages? National Institute Economic Review [J], 170, 87-98.

Sui, Y. H. (2004). Urban innovation system and urban innovation circle. Academic Circles [J], 106, $105-112$.

Tan, Y., O'connor, K., \& Westerman, C. (2008). The making of knowledge cities: Melbourne's knowledgebased urban development experience. Cities [J], 25, 63-72.

Tan, C. Y., Wei, J. H., Tian-Shu, X. U. et al. (2014). Urban sprawl and ecological environment dynamic of resource-based city__ A case study of Baotou. Forest Inventory and Planning [J], (01), 28-32.

2THINKNOW. (2019, Nov 13). Innovation Cities ${ }^{\mathrm{TM}}$ Index 2019: Globa [EB/OL]. https://www.innovationcities.com/index-2019-global-city-rankings/18842/

Viezzer, J., \& Biondi, D. (2021). The influence of urban, socio-economic, and eco-environmental aspects on COVID-19 cases, deaths and mortality: A multi-city case in the Atlantic Forest, Brazil. Sustainable Cities and Society [J], 69, 102859.

Wang, M. H., \& Yuh-Shan, H.-Z. (2019). Global performance and development on sustainable city based on natural science and social science research: A bibliometric analysis. The Science of the Total Environment [J], 666, 1245-1254.

Wang, J., Yang, Z., \& Qian, X. (2020). Driving factors of urban shrinkage: Examining the role of local industrial diversity. Cities [J], 99, 102646.

Wang, R. S., Li, F., Han, B. L., et al. (2014). Urban eco-complex and eco-space management. Acta Ecologica Sinica $[J], 34(1)$.

Wei, H. (2015). The administrative hierarchy and growth of urban scale in China. Chinese Journal of Urban and Environmental Studies [J]., 03, 1550001.

Wen, D. W. (2011). Economic scale, technological innovation and vertical specialization. The Journal of Quantitative \& Technical Economics [J], 28(8), 19.

Yang, S. Y., Zheng, L. I., Sun, G. Z., et al. (2019). Industrial development, urban expansion and innovative city construction: From the perspective of industry-city integration. Journal of Jiangxi University of Finance and Economics [J]., 01, 82. 
Yao, L., Liu, J., Wang, R., et al. (2015). A qualitative network model for understanding regional metabolism in the context of social-economic-natural complex ecosystem theory. Ecological Informatics [J], 26, 29-34.

Yong, Z., Shan, L., School, B., et al. (2016). Financial development, technical innovation and the construction of smart cities: An informatization development perspective. Journal of Finance and Economics $[J], 02,4-15$.

Yuan, B., \& Zhang, Y. (2020). Flexible environmental policy, technological innovation and sustainable development of China's industry: The moderating effect of environment regulatory enforcement. Journal of Cleaner Production [J], 243, 118543.

Zakaria, N. A., Ibrahim, S., Mahrin, M. N. (2016). Using grounded theory approach to identify value-based factors in software development[C]//2016 6th international conference on information and communication technology for The Muslim World (ICT4M).

Zhang, J., Chang, Y., Zhang, L., et al. (2018). Do technological innovations promote urban green development?-A spatial econometric analysis of 105 cities in China. Journal of Cleaner Production [J], 182, 395-403.

Zhang, H., Shen, L., Zhong, S., et al. (2020). Economic structure transformation and low-carbon development in energy-rich CITIES: The case of the contiguous area of Shanxi and Shaanxi provinces, and inner Mongolia autonomous region of China. Sustainability [J], 12, 1875.

Zhao, J., Hu Z. X. (2010). On structure and evolution of city innovation systems: agent-based modeling. Science of Science and Management of S. \& T. [J], (1), 7.

Zhao, L. M., Li, Z. H. (2003). The study of the city innovation system's dynamical rules. Studies in Science of Science [J], (1), 97-100.

Zheng, L. I., Yang, S., \& University, J. (2019). Has the pilot project of innovative cities increased the level of innovation? Economic Perspectives [J], 8, 70-85.

Zhou, Q., Liu, Z., Zhu, H., et al. (2012). The evolution of innovation system theory and its relations. Science of Science and Management of $S \& T[J], 33,50-55$.

Zhou, B., Zeng, X., Jiang, L., et al. (2020). High-quality economic growth under the influence of technological innovation preference in China: A numerical simulation from the government financial perspective. Structural Change and Economic Dynamics [J], 54, 163-172.

Zhou, R., Liu, Y., Yang, Z. (2019). Spatial-temporal evolution and knowledge spillovers of urban innovation in China. Economic Geography [J], 39(4), 8.

Publisher's Note Springer Nature remains neutral with regard to jurisdictional claims in published maps and institutional affiliations.

\section{Authors and Affiliations}

\section{Jing-Xiao Zhang ${ }^{1}$ (D) Jia-Wei Cheng ${ }^{1} \cdot$ Simon Patrick Philbin ${ }^{2}$. Pablo Ballesteros-Perez ${ }^{3} \cdot$ Martin Skitmore $^{4} \cdot$ Ge Wang $^{5}$}

Jia-Wei Cheng

chengjiawei0905@126.com

Simon Patrick Philbin

philbins@1sbu.ac.uk

Pablo Ballesteros-Perez

pabbalpe@dpi.upv.es

Martin Skitmore

Rm.skitmore@qut.edu.au

Ge Wang

ge_wang@yeah.net

1 School of Economics and Management, Chang'an University, 161 Changan Rd, Xian 710061, Shaanxi, People's Republic of China

2 Nathu Puri Institute for Eng. and Enterp., London South Bank University, 103 Borough Road, 


\section{London SE1 0AA, UK}

3 Departamento de Proyectos de Ingeniería, Dept Proyectos Ingn, Universitat Politècnica de València, Camino Vera S-N, 46009 Valencia, Spain

4 Faculty of Society and Design, Bond University, Gold Coast, QLD 4229, Australia

5 College of Public Administration, Huazhong Agricultural University, No. 1 Shizishan Street, Wuhan 430070, People's Republic of China 